\title{
Von toten Tanten und getauschten Grundstücken. Zur Bekämpfung der Regierungskorruption in Rumänien und der Rolle des Parlaments ${ }^{*}$
}

\author{
Michael Hein
}

Am 4. März 2009 beantragte das rumänische Parlament bei der Generalstaatsanwaltschaft des Landes die Eröffnung der Strafverfolgung gegen den früheren Premierminister Adrian Năstase. Mit diesem Beschluss fand ein mehr als zwei Jahre andauernder Konflikt über die Bekämpfung von Regierungskorruption sein vorübergehendes Ende. In diesem Konflikt hatte die überwiegende Mehrheit der politischen Elite mit allen Mitteln versucht zu verhindern, dass sich ehemalige und aktuelle Regierungsmitglieder vor Gericht in Strafverfahren verantworten müssen. Konkret waren acht Spitzenpolitiker von staatsanwaltschaftlichen Ermittlungen betroffen. Năstase, dem Korruptionsdelikte in Höhe von weit über einer Million Euro vorgeworfen werden, stilisierte sich angesichts der drohenden Anklage als unschuldiger, politisch verfolgter Kämpfer für die Beachtung von Recht und Gesetz:

„They say Năstase is not bold enough to prove his innocence, although it is obvious that it is not at all about guilt or innocence. They say I hide behind the Parliament, although the Constitution requires the Parliament's opinion on such things. [...] All these years [...] I have insisted that the Constitution and the other laws be respected. That is all. That is the only mistake I admit to having made all this time - that I wanted to fight for my principles and respect the laws of this country, although those who accuse me do not. "1

Die Ursache für die Auseinandersetzungen zwischen weiten Teilen der politischen Elite in Regierung und Parlament, reformfreudigen Kräften in Politik und Justiz, den Strafverfolgungsbehörden und dem Verfassungsgerichtshof lag bereits in der postsozialistischen Verfassung von 1991 begründet. Diese sieht für die Verfolgung von Straftaten, die Mitglieder der Regierung „bei der Ausübung ihres Amtes“ begangen haben, ein spezielles Verfahren vor: „Nur die Abgeordnetenkammer, der Senat und der Präsident Rumäniens haben das Recht, die Strafverfolgung [...] zu beantragen“" (Art. 109 Abs. 2 Verf.). ${ }^{2}$ Die Details werden per Gesetz geregelt. Damit versieht die Verfassung den Regierungschef und seine Minister mit einem stärkeren Schutz, als ihn die Mitglieder des rumänischen Zweikammerparlaments durch ihre Immunität genießen. Die Abgeordneten und Senatoren können zwar ohne die Zustimmung der jeweiligen Kammer „nicht durchsucht, festgehalten oder verhaftet werden“ (Art. 72, Abs. 1 Verf.); es ist jedoch uneingeschränkt möglich, gegen Parlamentsmitglieder zu ermitteln, Anklage zu erheben und sie gegebenenfalls zu verurteilen.

In Rumänien stehen daher die Mitglieder der Regierung in einem faktisch rechtsfreien Raum - jedenfalls solange es gleichgerichtete Mehrheitsverhältnisse in beiden Parlamentskammern sowie einen dem Regierungslager zugehörigen Präsidenten gibt und die politische

* Für Anregungen und Hinweise danke ich Hubertus Buchstein, Radu Harald Dinu, Stefan Ewert und Antonia Geisler sowie den Redaktionsmitgliedern der Zeitschrift für Parlamentsfragen.

1 Nine O'Clock (in Bukarest erscheinende englischsprachige Tageszeitung) vom 9. Februar 2009, S. 3 .

2 Hier in der Zählung nach der Verfassungsreform von 2003. Zitiert nach der Übersetzung von Axel Bormann, Die rumänische Verfassungsreform 2003. Übersetzung mit Einleitung, in: Jahrbuch für Ostrecht, 45. Jg. (2004), H. 1, S. $207-267$. 
Soziokultur der politischen Elite verhindert, dass eine Parlamentskammer oder das Staatsoberhaupt Strafverfolgungsanträge gegen die „eigenen Leute“ stellen. ${ }^{3}$ Für einen solchen Schutzraum fehlt jedoch im Gegensatz zur parlamentarischen Immunität jede plausible Begründung. Lässt sich diese im Sinne eines Schutzes der Legislative vor Übergriffen der Exekutive im Rahmen einer gewaltenteilenden Verfassungsordnung legitimieren, liegt bei einem vergleichbaren oder gar noch stärkeren Schutz der Exekutive nur der „Verdacht einer Selbstbegünstigung der politischen Elite “4 nahe - auch wenn solche Regelungen in vielen europäischen Ländern anzutreffen sind. ${ }^{5}$ Dies gilt insbesondere dann, wenn wie in Rumänien die Strafverfolgungsbehörden der Exekutive unterstellt sind und daher kaum für die politischen Ziele der Opposition nutzbar gemacht werden können. Umgekehrt ist es jedoch sowohl der Regierungsmehrheit als auch der parlamentarischen Opposition gerade deshalb möglich, Korruptionsvorwürfe politisch zu instrumentalisieren.

Politische Korruption steht seit geraumer Zeit im Fokus politikwissenschaftlicher Forschung. ${ }^{6}$ Dies gilt insbesondere für die Länder Ost- und Südosteuropas, in denen sie seit dem Beginn der politischen und ökonomischen Transformationen Anfang der 1990er Jahre vielfach als virulentes Problem wahrgenommen wird. Zum einen wurden hier die Ursachen politischer Korruption untersucht und in den spezifischen Umständen der Transformation, der Politischen Kultur und gerade mit Blick auf Südosteuropa im Vorhandensein klientelistischer Strukturen gesehen. 7 Zum anderen wird häufig auf ihre Gefahren hingewiesen. Diese werden vor allem in den jeweils betroffenen Ländern verortet. Dort behindert Korruption die wirtschaftliche Entwicklung, gefährdet den demokratischen und rechtsstaatlichen Charakter der Regierungssysteme, und diese geraten in zum Teil gravierende Vertrau-

3 „Politische Soziokultur“ hier im Sinne von Karl Rohe, Politische Kultur: Zum Verständnis eines theoretischen Konzepts, in: Oskar Niedermayer / Klaus von Beyme (Hrsg.), Politische Kultur in Ost- und Westdeutschland, Berlin 1994, S. 1 - 21. Vgl. zur Politischen Kultur Rumäniens Richard Andrew Hall, Political Culture in Post-Ceauşescu Romania, in: Henry F. Carey (Hrsg.), Romania since 1989. Politics, Economics, and Society, Lanham u.a. 2004, S. 215 - 225; Tina Olteanu, Korruption in Rumänien - ein Erbe des Staatssozialismus?, in: Dieter Segert (Hrsg.), Postsozialismus. Hinterlassenschaften des Staatssozialismus und neue Kapitalismen in Europa, Wien 2007, S. $65-85$.

4 Julia Wuttke, Die Verantwortlichkeit von Regierungsmitgliedern in Deutschland und Frankreich, Köln u.a. 2005, S. 205.

5 So gewährt beispielsweise mehr als ein Drittel der EU-Mitgliedstaaten ihren Regierungsmitgliedern einen vergleichbaren Schutz; neben Rumänien sind dies Belgien, Estland, Frankreich, Griechenland, Italien, Litauen, die Niederlande, Polen und Portugal, vgl. Astrid Epiney / Ulrich Hufeld, Europäisches Verfassungsrecht. Textsammlung, Baden-Baden 2009. Siehe zu den einzelnen Verfassungen, http://www.verfassungen.de (Abruf am 13. März 2009).

6 Vgl. die einschlägigen Sammelwerke der vergangenen Jahre: Themenschwerpunkt Korruption, in: APuZ, B 3 / 4 (2009); Ulrich von Alemann (Hrsg.), Dimensionen politischer Korruption. Beiträge zum Stand der internationalen Forschung, in: PVS, Sonderheft 35, Wiesbaden 2005; Harald Bluhm / Karsten Fischer (Hrsg.), Sichtbarkeit und Unsichtbarkeit der Macht. Theorien politischer Korruption, Baden-Baden 2002; Arnold J. Heidenheimer / Michael Johnston (Hrsg.), Political Corruption: Concepts and Contexts, New Brunswick u.a. 2002.

7 Vgl. Iuliana Precupetu, On the Nature and Causes of Corruption in Romania. Evidence From a Grounded Theory Approach, Discussion Paper, University of Konstanz 2008, S. 17 ff., http://www. uni-konstanz.de/crimeandculture/docs/Discussion_Paper_No_13_ICCV_April_2008.pdf (Abruf am 22. September 2009); Plamen K. Georgiev, Corruptive Patterns of Patronage in South East Europe, Wiesbaden 2008; William L. Miller / Ase B. Grødeland / Tatyana Y. Koshechkina, A Culture of Corruption? Coping with Government in Post-Communist Europe, Budapest u.a. 2001. 
enskrisen gegenüber ihren Bevölkerungen. ${ }^{8}$ Grassierende Korruption in EU-Mitgliedstaaten bedroht aber auch die Stabilität und das Funktionieren der Union selbst. In diesem Kontext sind die Bedingungen der Strafverfolgung von Regierungskorruption von zentraler Bedeutung. Im Falle Rumäniens stellt sich dabei eine ganz grundlegende Frage: Sind Politiker bereit, ihre Tätigkeit von unabhängigen Organen der Justiz auf strafrechtliche Relevanz prüfen zu lassen und sich gegebenenfalls einem gerichtlichen Verfahren wegen Korruptionsvergehen zu unterziehen? Die Antwort fällt weitgehend negativ aus, wie die folgende Analyse des Verfassungskonflikts um die strafrechtliche Verfolgung aktueller und ehemaliger Regierungsmitglieder in Rumänien von Ende 2006 bis zum Regierungswechsel im Oktober 2009 zeigt.

\section{Die Ausgangslage: Das Gesetz über die Ministerielle Verantwortlichkeit}

Das bereits 1991 von Art. 109 Verf. geforderte Gesetz über die Ministerielle Verantwortlichkeit (MVG) war erst siebeneinhalb Jahre nach der Verfassunggebung im April 1999 verabschiedet und in den Folgejahren mehrfach geändert und ergänzt worden. ${ }^{9}$ Vor Ausbruch des hier behandelten Konflikts beinhaltete es folgende wichtige Regelungen:

- Ein Antrag auf Verfolgung von Straftaten, die Mitglieder der Regierung in Ausübung ihres Amtes begangen hatten, musste von der zuständigen Staatsanwaltschaft beim Staatspräsidenten oder einer der beiden Parlamentskammern gestellt werden; die Abgeordnetenkammer beziehungsweise der Senat entschieden über einen solchen Antrag in geheimer Abstimmung (Art. $15 \mathrm{MVG}$ ).

- Beim Amt des Staatspräsidenten wurde eine Kommission zur Überprüfung allfälliger Strafverfolgungsanträge eingerichtet, der auf Vorschlag des Justiz- und des Innenministers fünf Mitglieder angehörten. Der Präsident konnte über einen Antrag auf Strafverfolgung erst nach der Bearbeitung durch diese Kommission entscheiden. Bei Regierungsmitgliedern, die zugleich Abgeordnete oder Senatoren sind, sollte anschließend zusätzlich zum Staatspräsidenten die jeweilige Parlamentskammer dem Ersuchen der Staatsanwaltschaft zustimmen müssen (Art. $16 \mathrm{MVG}$ ).

- Ein von der Abgeordnetenkammer, dem Senat oder dem Staatspräsidenten bewilligter Antrag hatte anschließend an den Justizminister weitergeleitet zu werden, der „gemäß des Gesetzes“"weiter verfahren sollte (Art. 18 MVG).

- Der Geltungsbereich des Gesetzes umfasste nicht nur die aktuellen, sondern auch alle ehemaligen Mitglieder der Regierung (Art. $20 \mathrm{MVG}$ ).

Mit diesen Bestimmungen erweiterte das Gesetz über die Ministerielle Verantwortlichkeit den Schutzraum der Regierungsmitglieder nochmals deutlich über die Vorgaben der Verfas-

8 Vgl. Ivana Marková (Hrsg.), Trust and Democratic Transition in Post-communist Europe, Oxford u.a. 2004; Iuliana Precupetu, Corruption in Romania. First Steps Towards a Grounded Theory of Corruption, Discussion Paper, University of Konstanz 2007, S. 26 f., http://www.unikonstanz.de/crimeandculture/docs/Discussion_Paper_No_4_ICCV_Romania_July_2007.pdf (Abruf am 22. September 2009); Michael Hein, Die Europa- und Parlamentswahlen 2009 in Bulgarien, in: Südosteuropa Mitteilungen, 49. Jg. (2009), H. 5, S. 44 - 61, S. 48 ff.

9 Gesetz Nr. 115/1999. Ursprüngliche Fassung in: Monitorul Oficial al României (Amtsblatt MO) 300/1999; nach Überarbeitungen und Ergänzungen erneut veröffentlicht in: MO $334 / 2002$. 
sung hinaus. Zum einen wurde die Regierung selbst mehrfach in das Verfahren mit einbezogen. Zum anderen wurden nun auch alle ehemaligen Regierungsmitglieder durch das MVG protegiert, obwohl für diese keinerlei Schutzinteresse für die laufende Arbeit der Exekutive geltend gemacht werden konnte.

Zu einem Konflikt über die Schutzregelungen des Art. 109 Verf. und des MVG sollte es jedoch erst nach dem Regierungswechsel infolge der Parlaments- und Präsidentschaftswahlen vom November/Dezember 2004 kommen. Zum neuen Staatsoberhaupt wurde dabei der bisherige Vorsitzende der Demokratischen Partei (Partidul Democrat - PD), Traian Băsescu, gewählt, der die Präsidentschaftswahl knapp gegen Adrian Năstase, den amtierenden Premierminister und Vorsitzenden der Sozialdemokratischen Partei (Partidul Social Democrat - PSD) gewann. Die PSD-Regierung, die zeitweilig mit der Humanistischen Partei (Partidul Umanist Român - PUR) koaliert hatte und im Parlament vom Demokratischen Verband der Ungarn Rumäniens (Uniunea Democrată Maghiară din România UDMR) unterstützt worden war, verlor ebenfalls ihre Mehrheit. Die neue Regierung wurde aus vier Koalitionspartnern gebildet: Die Nationalliberale Partei (Partidul Național Liberal - PNL) und die Demokratische Partei, die gemeinsam in einer Listenverbindung kandidiert hatten, lösten den Ungarnverband und die PUR aus ihrer Zusammenarbeit mit der PSD heraus und konnten so knappe Mehrheiten in beiden Parlamentskammern erringen. Neuer Premierminister wurde der PNL-Vorsitzende Călin Popescu-Tăriceanu. ${ }^{10}$

Nationalliberale und Demokraten waren unter anderem mit dem Ziel angetreten, erstmals nach 1990 die grassierende Korruption in Rumänien ernsthaft zu bekämpfen und dabei insbesondere die Korruption auf hoher politischer Ebene wirksam zu verfolgen. Zur Protagonistin dieser Bemühungen wurde die neue Justizministerin Monica Macovei. Die frühere Staatsanwältin war als parteilose Vertreterin der "Zivilgesellschaft“ auf Wunsch der Demokratischen Partei und insbesondere ihres bisherigen Vorsitzenden Băsescu in die Regierung gelangt, um damit die parteipolitische Unabhängigkeit der neuen Rechtspolitik zu symbolisieren. Bis 2004 war Macovei Vorsitzende des Helsinki-Komitees in Rumänien und als Rechtsanwältin beim Europäischen Gerichtshof für Menschenrechte tätig gewesen. Das neue Regierungsbündnis zerbrach jedoch bereits im Frühjahr 2007. Zum einen saßen PNL und PD mit zentralen Protagonisten ihres bisherigen politischen Gegners in einem Boot, die sich bis zum Regierungswechsel allen erfolgversprechenden Bemühungen um eine erfolgreiche Korruptionsbekämpfung widersetzt hatten. Dies betraf insbesondere die Humanistische Partei - 2005 umbenannt in Konservative Partei (Partidul Conservator - PC) -, die schon im Dezember 2006 die Koalition verließ. Zum anderen kristallisierte sich rasch eine scharfe Konkurrenzsituation zwischen Staatspräsident Băsescu und Premierminister Popescu-Tăriceanu beziehungsweise zwischen PNL und PD heraus, die schließlich im Frühjahr 2007 zum Auseinanderbrechen der Koalition und zur Unterstützung eines erfolglosen Amtsenthebungsverfahrens gegen Băsescu seitens der Nationalliberalen führte (vgl. Tabelle 1). Das Verhältnis von Băsescu und Popescu-Tăriceanu war seitdem von tiefem Misstrauen und regelmäßigen gegenseitigen Angriffen geprägt, die auch den hier untersuchten Konflikt vielfach überlagerten.

10 Vgl. William M. Downs / Raluca V. Miller, The 2004 Presidential and Parliamentary Elections in Romania, in: Electoral Studies, 25. Jg. (2006), H. 2, S. 409 - 415; Sorana Pârvulescu, 2004 Romanian Elections: Test Case for a True Romanian Democracy, in: Romanian Journal of Political Science, 4. Jg. (2004), H. 2, S. 7 - 28. 


\begin{tabular}{|l|l|l|l|l|}
\hline \multicolumn{2}{|c|}{ Tabelle 1: Die rumänischen Regierungen seit Ende 2004} \\
\hline Zeitraum & $\begin{array}{l}\text { Regierungs- } \\
\text { parteien }\end{array}$ & Art der Regierung & Premierminister & \multicolumn{1}{|c|}{ Justizminister } \\
\hline $\begin{array}{l}12.2004- \\
12.2006\end{array}$ & $\begin{array}{l}\text { PNL, PD, } \\
\text { UDMR, } \\
\text { PUR/PC }\end{array}$ & Mehrheitsregierung & $\begin{array}{l}\text { Călin Popescu- } \\
\text { Tăriceanu (PNL) }\end{array}$ & $\begin{array}{l}\text { Monica Macovei } \\
\text { (parteilos) }\end{array}$ \\
\hline $\begin{array}{l}12.2006- \\
04.2007\end{array}$ & $\begin{array}{l}\text { PNL, PD, } \\
\text { UDMR }\end{array}$ & Minderheitsregierung & $\begin{array}{l}\text { Călin Popescu- } \\
\text { Tăriceanu (PNL) }\end{array}$ & $\begin{array}{l}\text { Monica Macovei } \\
\text { (parteilos) }\end{array}$ \\
\hline $\begin{array}{l}04.2007- \\
12.2008\end{array}$ & $\begin{array}{l}\text { PNL, } \\
\text { UDMR }\end{array}$ & Minderheitsregierung & $\begin{array}{l}\text { Călin Popescu- } \\
\text { Tăriceanu (PNL) }\end{array}$ & $\begin{array}{l}\text { Tudor Chiuariu } \\
\text { (PNL, bis 01.2008), } \\
\text { Teodor Melescanu } \\
\text { (PNL, 01./02.2008), } \\
\text { Cătălin Predoiu } \\
\text { (PNL, ab 02.2008) }\end{array}$ \\
\hline $\begin{array}{l}12.2008- \\
10.2009\end{array}$ & $\begin{array}{l}\text { PD-L, PSD, } \\
\text { PC }\end{array}$ & Mehrheitsregierung & Emil Boc (PD-L) & $\begin{array}{l}\text { Cătălin Predoiu } \\
\text { (parteilos) }\end{array}$ \\
\hline Quelle: Eigene Zusammenstellung. & \\
\hline
\end{tabular}

In den ersten Regierungsmonaten wurden die Reformbemühungen der Justizministerin jedoch noch von allen Koalitionspartnern unterstützt. Eines ihrer Vorhaben betraf das Gesetz über die Ministerielle Verantwortlichkeit. Am 27. Januar 2005 modifizierte die Regierung das Gesetz per Dringlichkeitsanordnung dahingehend, dass es ausschließlich für amtierende Regierungsmitglieder gelte, während für eine Strafverfolgung ehemaliger Premierminister und Minister die allgemeinen Bestimmungen der Strafprozessordnung Anwendung finden sollten (Art. 23 Abs. 2 f. MVG). ${ }^{11}$ Diese Änderung wurde bis Ende März 2005 von beiden Parlamentskammern mit breiten Mehrheiten bestätigt. ${ }^{12}$ Damit kam die rumänische Politik auch einer der zentralen Forderungen nach, die die Europäische Kommission in ihren Fortschrittsberichten vor dem EU-Beitritt regelmäßig erhoben hatte. ${ }^{13}$

Darüber hinaus wurden die Justizorganisationsgesetze bis Mitte 2005 umfangreich reformiert. ${ }^{14}$ In diesem Zusammenhang wurde insbesondere die bisher weitgehend ineffek-

11 Dringlichkeitsanordnung Nr. 3/2005, in: MO 116/2005. Die hier angegebene Artikelzählung bezieht sich auf die konsolidierte Fassung des MVG in: MO 200/2007. Die rumänische Regierung hat laut Art. 115 Abs. 4 ff. Verfassung das Recht, in ,außergewöhnlichen Fällen, in denen die Regelung nicht hinausgeschoben werden kann", solche unmittelbar in Kraft tretenden Dringlichkeitsanordnungen zu erlassen. Diese werden an das Parlament weitergeleitet und können dort als Gesetz beziehungsweise Gesetzesänderung angenommen, geändert oder abgelehnt werden. Von ihrem Dringlichkeitsanordnungsrecht macht die Regierung Rumäniens seit Mitte der 1990er Jahre in sehr großem Umfang Gebrauch.

12 Gesetz Nr. 90/2005, in: MO 322/2005; Allgemeine Deutsche Zeitung für Rumänien (Bukarest) vom 23. Februar 2005, S. 1.

13 Vgl. zuletzt Kommission der Europäischen Gemeinschaften, Regelmäßiger Bericht über die Fortschritte Rumäniens auf dem Weg zum Beitritt, Brüssel, 6. Oktober 2004, S. 23, http://ec.europa. eu/enlargement/archives/pdf/key_documents/2003/rr_ro_final_de.pdf (Abruf am 12. Februar 2009).

14 Dabei wurden jedoch schon die ersten Risse im Verhältnis der Regierungsparteien sichtbar, die kurzfristig zu einer ersten ernsthaften Regierungskrise führten. Vgl. Anneli Ute Gabanyi, Verfassungskrise in Rumänien, Diskussionspapier der Stiftung Wissenschaft und Politik, Berlin 2005; Günther H. Tontsch, Rumänien: Verfassungsgericht verwässert Justizreform und verursacht Regierungskrise, in: WGO-Monatshefte für osteuropäisches Recht, 47. Jg. (2005), H. 3, S. 166 - 168. 
tive Antikorruptionsstaatsanwaltschaft (ab 2006: Antikorruptionsdirektion) in eine effektive Strafverfolgungsbehörde umgewandelt, die sich nun erstmals auch intensiv der Korruption auf höchster politischer Ebene zuwandte. Eine wirksame Bekämpfung der politischen Korruption hatte es zuvor weder unter den sozialistischen beziehungsweise sozialdemokratischen Regierungen (1990 bis 1996 und 2000 bis 2004) noch während der Regierungszeit einer Mitte-Rechts-Koalition (1996 bis 2000) gegeben.

\section{Die Affäre Năstase und das erste MVG-Urteil des Verfassungsgerichtshofes}

Im Frühjahr 2006 erreichte die bereits seit 1999 brodelnde Korruptionsaffäre um den früheren Premierminister und aktuellen Präsidenten der Abgeordnetenkammer, den PSD-Vorsitzenden Adrian Năstase ihren vorläufigen Höhepunkt. Gegen ihn waren nach und nach schwerwiegende Vorwürfe vorgebracht worden. Diese betrafen insbesondere eine dubiose Erbschaft in Höhe von ca. 400.000 Euro, die Năstases Ehefrau Dana von einer „Wahltante“, einer Georgierin namens Tamara, geerbt haben wollte. Angeblich hatte „Tante Tamara ihr Vermögen vor allem mit Grundstücksspekulationen in den ersten Jahren der marktwirtschaftlichen Transformation Rumäniens verdient. Verwunderlich an dieser Geschichte war neben der Wahlverwandtschaft jedoch, dass die mysteriöse Tante trotz ihres Reichtums bis zu ihrem Tod zurückgezogen in einer kleinen Plattenbauwohnung in Bukarest gelebt hatte. Ein anderer Vorwurf bezog sich auf den Kauf eines Grundstücks im Zentrum der Hauptstadt, für das Adrian Năstase einem mittlerweile wegen Korruptionsverbrechen in Haft sitzenden Parteifreund den ungewöhnlich niedrigen Preis von 11.000 Euro gezahlt hatte, obwohl der reale Wert des Grundstücks auf das 25-fache geschätzt wurde. Darüber hinaus wurde Năstase beispielsweise der Annahme von Bestechungsgeldern in Höhe von etwa 100.000 Euro sowie der illegalen Finanzierung seiner Präsidentschaftswahlkampagne 2004 beschuldigt. ${ }^{15}$ Am 15 . März 2006 musste er schließlich nach starkem öffentlichen und innerparteilichen Druck vom Vorsitz der Abgeordnetenkammer und sämtlichen Parteiämtern zurücktreten. ${ }^{16} \mathrm{Im}$ November 2006 erhob die Antikorruptionsdirektion Anklage gegen ihn und seine Ehefrau wegen Amtsmissbrauchs, Korruption und Bestechung in einigen dieser Fälle, bei denen sie sich insgesamt ca. 1,37 Millionen Euro illegal angeeignet haben sollen. ${ }^{17}$

Damit begann der Konflikt um die Möglichkeit zur Bekämpfung von Regierungskorruption. Năstase klagte im Rahmen seines Strafverfahrens über den Weg einer konkreten Normenkontrolle vor dem Verfassungsgerichtshof gegen die Änderung des Gesetzes über die Ministerielle Verantwortlichkeit, mit der ehemalige Minister aus dem Geltungsbereich des MVG herausgenommen worden waren. In seiner ersten MVG-Entscheidung vom 5. Juli 2007 legte das Gericht Art. 109 Verf. zwar dahingehend aus, dass er sich unzweifelhaft ausschließlich auf amtierende Minister beziehe, was sich bereits aus der verwendeten Terminologie ergebe - im Verfassungstext ist von „Regierungsmitglieder[n]“, nicht von früheren

15 Handelsblatt Global Reporting vom 17. Januar 2006, http://blog.handelsblatt.de/warschau-vetter/eintrag.php?id=9 (Abruf am 10. März 2009); Der Standard vom 20. November 2006, 20. Januar 2009 und 12. März 2009, http://derstandard.at/?url=/?id=2665360, http://derstandard. at/?url=/?id=1231152410368 und http://derstandard.at/?url=/?id=1234509228061 (Abruf am 12. März 2009).

16 Allgemeine Deutsche Zeitung für Rumänien vom 16. März 2006, S. 1.

17 Allgemeine Deutsche Zeitung für Rumänien vom 15. November 2006, S. 1. 
Amtsinhabern die Rede. Gleichwohl wurde Art. 23 Abs. 2 f. MVG als verfassungswidrig verworfen. Das Gericht befand, dass „to exclude former Members of Government [...] from the special derogatory procedure provided for Members of Government, according to a random criterion, established according to the moment of initiation of the procedure, during the mandate or after cessation thereof," den Grundsatz der Gleichheit vor dem Gesetz (Art. 16 Verf.) verletze. ${ }^{18}$ Diese selbstwidersprüchliche Interpretation stieß auf nachvollziehbare Kritik der Rechtswissenschaft: „Es leuchtet juristisch nicht unmittelbar ein, warum das Verfassungsgericht einerseits ausdrücklich erklärt, dass sich der Schutz des Artikel 109 nur auf amtierende Minister bezieht und damit zugesteht, dass die Situation amtierender Minister nicht mit der ehemaliger zu vergleichen ist, andererseits aber eine Verletzung des Gleichheitsgrundsatzes feststellt. "19 Wenn überhaupt, dann hätte der Verfassungsgerichtshof aufgrund eines Widerspruchs innerhalb der Verfassung zwischen den Artikeln 16 und 109 „verfassungswidriges Verfassungsrecht“ diagnostizieren müssen, um im Ergebnis dem rechtsstaatlichen Grundsatz der Gleichheit vor dem Gesetz den Vorrang gegenüber den Vorschriften zur ministeriellen Verantwortlichkeit geben zu können. Soweit wollte das Gericht aber offenbar nicht gehen. Stattdessen stellte es mit einer wenig überzeugenden Interpretation der Verfassung sämtliche ehemalige Regierungsmitglieder zurück unter den Schutzschirm des MVG. Allerdings kam diese Entscheidung nicht überraschend: Alle sieben der an dem Urteil beteiligten Richter waren ehemalige PSD-Minister und/oder -Parlamentarier oder konnten aus anderen Gründen der PSD parteipolitisch zugeordnet werden. ${ }^{20}$

Dadurch wurde das Strafverfahren gegen Adrian Năstase zunächst hinfällig, da die Ermittlungen der Antikorruptionsdirektion nunmehr nicht verfassungskonform initiiert worden waren. Năstase war jedoch zu diesem Zeitpunkt bei weitem nicht das einzige ehemalige oder aktuelle Regierungsmitglied, gegen das die Antikorruptionsdirektion beziehungsweise die Direktion zur Ermittlung von Straftaten des Organisierten Verbrechens und des Terrorismus ermittelte ${ }^{21}$ : (1) Ex-Transportminister Miron Mitrea (2000 bis 2004, PSD) wurde die Annahme von Bestechungsgeldern für die Vergabe von Aufträgen im Straßenbau und die Anstiftung zur Fälschung amtlicher Dokumente vorgeworfen. (2) Der ehemalige Wirt-

18 Verfassungsgerichtsentscheidung Nr. 665/2007, in: MO 547/2007, Englische Übersetzung, S. 7, http://www.ccr.ro/decisions/pdf/en/2007/D0665_07.pdf (Abruf am 10. März 2009).

19 Stefanie Ricarda Roos, Bahn frei für die strafrechtliche Ermittlung politischer Korruptionsfälle in Rumänien?, Konrad Adenauer Stiftung, Bukarest, 29. Januar 2008, S. 4, http://www.kas.de/ rspsoe (Abruf am 8. April 2008).

20 So etwa Şerban Viorel Stănoiu, der 2001 als Ehemann der damaligen PSD-Justizministerin von Staatspräsident Ion Iliescu zum Verfassungsrichter ernannt worden war. Vgl. zum Bestellungsmodus der Richter, der einen hohen parteipolitischen Einfluss der Parlamentsmehrheiten und des Staatspräsidenten ermöglicht, Vasile Gionea / Günther H. Tontsch, Die Verfassungsgerichtsbarkeit in Rumänien, in: Otto Luchterhandt / Christian Starck / Albrecht Weber (Hrsg.), Verfassungsgerichtsbarkeit in Mittel- und Osteuropa 1: Berichte, Baden-Baden 2007, S. 105 - 127, S. 108.

21 Beide Direktionen sind an der Generalstaatsanwaltschaft des höchsten rumänischen Gerichts, des Hohen Kassations- und Gerichtshofes, angesiedelt. Vgl. im Folgenden Stefanie Ricarda Roos, Unterschiedliche Ampelstellung für Eröffnung strafrechtlicher Ermittlungsverfahren gegen hochrangige rumänische Politiker, Konrad Adenauer Stiftung, Bukarest, 28. August 2008, http://www. kas.de/rspsoe (Abruf am 10. März 2009); Radio Romania International vom 20. September 2007, http://www.rri.ro/arh-art.shtml?lang=7\&sec=279\&art=5549 (Abruf am 10. März 2009); Allgemeine Deutsche Zeitung für Rumänien vom 20. September und 12. Oktober 2007, S. 1; Nine O'Clock vom 17. Juli 2008, S. 1. 
schaftsminister Codruț Şereş (2004 bis 2006, PUR/PC) wurde der Preisgabe von Wirtschaftsgeheimnissen im Zusammenhang mit zwei größeren Privatisierungsvorhaben beschuldigt. (3) Ex-Verteidigungsminister Victor Babiuc (1996 bis 2000, damals PD, später PNL und erneut PD) wurde Amtsmissbrauch und die Annahme von Bestechungsgeldern vorgeworfen. Dies betraf insbesondere einen undurchsichtigen Grundstückstausch des Verteidigungsministeriums mit dem zwielichtigen Geschäftsmann George „Gigi“ Becali aus dem Jahr 1999. Dieser hatte für ein relativ preisgünstiges Grundstück von 21 Hektar Größe in einem Dorf nahe Bukarest eine gleich große Fläche in Pipera am Nordrand der Hauptstadt erhalten, wo in den Folgejahren zahlreiche Bürogebäude, Luxushotels und Wohnhäuser entstanden. Das „ertauschte“ Grundstück soll inzwischen einen Marktwert von 200 Millionen Euro haben. Becali, der mittlerweile mit seiner eigenen Christlich-Demokratischen Partei der Neuen Generation (Partidul Noua Generaţie - Creştin şi Democrat - PNG-CD) auch in der Politik Fuß gefasst hat und im Juni 2009 ins Europäische Parlament gewählt wurde, baute auf dieser Transaktion seinen späteren Reichtum auf, mit dem er unter anderem den in seinem Besitz befindlichen Fußballverein Steaua Bukarest finanziert. $^{22}$ (4) Arbeitsminister Paul Păcuraru (2007/2008, PNL) wurde der Bestechlichkeit zugunsten eines seiner Familienangehörigen bei der Vergabe staatlicher Aufträge beschuldigt. (5) Auch gegen Landwirtschaftsminister Decebal Traian Remeş (2004 bis 2007, PNL) bestand der Verdacht der Bestechlichkeit, der sich am 11. Oktober 2007 bestätigte, als im Fernsehen Filmaufnahmen veröffentlicht wurden, die ihn bei der Annahme von 15.000 Euro Bestechungsgeld zeigen. (6) Gegen den ehemaligen Telekommunikationsminister Zsólt Nagy (2004 bis 2007, UDMR) wurde in einem Korruptions- und Spionage-Skandal ermittelt. Er soll eine internationale kriminelle Gruppierung unterstützt und Staatsgeheimnisse verraten haben. (7) Ermittelt wurde auch gegen den Nachfolger Monica Macoveis, den erst seit April 2007 amtierenden Justizminister Tudor Chiuariu (2007, PNL), wegen der Privatisierung eines Grundstücks der rumänischen Post, bei der er sich des Amtsmissbrauchs schuldig gemacht haben soll. In diesen Fall war auch Zsólt Nagy verwickelt.

Diese Liste gibt einerseits einen erschreckenden Einblick in das umfangreiche Ausmaß der Korruption in den Spitzen der rumänischen Politik - und sie umfasst ja „nur“ Verdächtige unter den ehemaligen und aktuellen Regierungsmitgliedern. Andererseits wird aber auch deutlich, dass erstmals nach 1991 gegen hochrangige Politiker aller politischen Lager ermittelt wurde.

\section{Die „Antikorruptionspolitik“ der PNL-UDMR-Minderheitsregierung und das zweite MVG-Urteil}

Gleichwohl wurde der Generalstaatsanwaltschaft Rumäniens und insbesondere der bei ihr angesiedelten Antikorruptionsdirektion von Seiten der PSD, der PC und des UDMR und in zunehmendem Maße auch der PNL immer wieder vorgeworfen, Parteiinteressen der PD beziehungsweise des Staatspräsidenten Traian Băsescu zu vertreten. Damit begründeten die

22 Vgl. Keno Verseck, Vom Schafhirten zum Milliardär. Gigi Becali - eine rumänische Karriere, Deutschlandfunk vom 15. April 2008, http://www.dradio.de/download/83439/ (Abruf am 12. März 2009); Website des Europäischen Parlaments, http://www.europarl.europa.eu/parliament/ archive/elections2009/en/romania_en.html (Abruf am 21. September 2009). 
Gegner einer wirksamen Korruptionsbekämpfung - namentlich Adrian Năstase - ihren Widerstand gegen eine strafrechtliche Verfolgung möglicher Korruptionsdelikte. Dieser Vorwurf vermochte jedoch aus zwei Gründen nicht recht zu überzeugen: Zum einen ermittelte die Antikorruptionsdirektion durchaus auch gegen Mitglieder der Demokratischen Partei, wie das Beispiel Victor Babiuc zeigt. Zum anderen wäre die Staatsanwaltschaft mit unbegründeten, aus rein politischen Interessen erhobenen Anklagen vor Gericht gescheitert, denn die rumänischen Richter können seit der Justizreform von 2004 nicht mehr von der Exekutive durch eine faktische Kontrolle des Obersten Rates der Magistratur (ORM), dem Selbstverwaltungsorgan der Judikative, verfassungswidrig beeinflusst werden, wie es bis dahin möglich gewesen war. ${ }^{23}$

Seit April 2007 regierte jedoch eine PNL-UDMR-Minderheitsregierung, die im Parlament von der Unterstützung der Sozialdemokratischen und der Konservativen Partei abhängig war. Schon seit Mitte 2005 hatte sich zudem gezeigt, dass PNL und UDMR immer weniger die Fortschritte im Bereich der Korruptionsbekämpfung unterstützten, die die Strukturreformen der Justizministerin Macovei nach und nach zeitigten. Dieser Trend verstärkte sich noch mit dem Beitritt Rumäniens zur Europäischen Union am 1. Januar 2007, da die EU-Kommission nach diesem Termin über keine ernsthaften Sanktionsmittel mehr gegenüber dem Neumitglied verfügte - jedenfalls über keine, die auch nur annähernd soviel Druck hätten aufbauen können wie noch während der Vorbeitrittsphase. Darüber hinaus standen mit Tudor Chiuariu und Paul Păcuraru auch zwei aktuelle Minister auf der oben aufgeführten Liste der Antikorruptionsdirektion.

Die neue Regierung ging nun offensiv daran, die Direktion zu schwächen und eine wirkungsvolle Korruptionsbekämpfung zu behindern. So versuchte der neue Justizminister Chiuariu zum einen mehrfach, die führenden Staatsanwälte der Antikorruptionsdirektion mit fadenscheinigen Begründungen auszutauschen. Er scheiterte mit diesem Vorhaben jedoch am Obersten Rat der Magistratur sowie an Präsident Băsescu. ${ }^{24}$ Zum anderen wurde die Regierung Popescu-Tăriceanu nun auch auf dem Gebiet der ministeriellen Verantwortlichkeit aktiv. Am 4. Oktober 2007 änderte sie per Dringlichkeitsanordnung das MVG dahingehend, dass die Präsidialkommission zur Überprüfung staatsanwaltschaftlicher Anträge auf Strafverfolgung zukünftig aus fünf vom ORM zu wählenden Richtern bestehen sollte (Art. $16 \mathrm{MVG}$ ). ${ }^{25}$ Zur Begründung hieß es, eine auf diese Weise zusammengesetzte Kommission könne die Ermittlungsakten objektiv prüfen. Gerade dies erschien jedoch völlig unplausibel: Warum sollten ausgerechnet Richter die Vorprüfung staatsanwaltschaftli-

23 Vgl. Camelia Toader, Korruptionsbekämpfung und Justizreform in Rumänien, in: WGO-Monatshefte für osteuropäisches Recht, 46. Jg. (2004), H. 4, S. 251 - 265; Moritz Hildebrand, Korruptionsbekämpfung in Rumänien. Gesetzeslage und Entwicklung 2005-2007, in: Osteuropa-Wirtschaft, 53. Jg. (2008), H. 3, S. 267 - 299; Axel Bormann, Rumänien: Rechtsrahmen und Institutionen, in: Herbert Küpper (Hrsg.), Korruptionsbekämpfung in Osteuropa. Berichte zu Ungarn, Kroatien, Bulgarien, der Slowakischen und der Tschechischen Republik, Polen und Rumänien, München 2009, S. 185 - 243.

24 Vgl. Stefanie Ricarda Roos, Aller guten Dinge sind für die rumänische Justiz nicht drei. Staatspräsident Basescu lehnt Ernennung national-liberaler Senatorin zur Justizministerin ab, Konrad Adenauer Stiftung, Bukarest, 10. Januar 2008, S. 1 f., http://www.kas.de/rspsoe (Abruf am 8. April 2008).

25 Dringlichkeitsanordnung Nr. 95/2007, in: MO 678/2007; Nine O'Clock vom 8. Oktober 2007, S. 3 . 
cher Ermittlungen übernehmen? Staatspräsident Băsescu warf Justizminister Chiuariu daraufhin vor, die Dringlichkeitsanordnung in eigenem Interesse verfasst zu haben, um den Kampf gegen die Korruption langfristig zu blockieren. ${ }^{26}$

Die Juristenvereinigungen Rumäniens protestierten gegen diese Gesetzesänderung als unvereinbar mit der richterlichen Unabhängigkeit und riefen die Richter zum Boykott der neuen Präsidialkommission auf. Volksanwalt Ioan Muraru reichte zudem auf Anregung dieser Vereinigungen wenige Tage nach dem Regierungserlass Klage vor dem Verfassungsgerichtshof ein. ${ }^{27}$ Muraru klagte dabei nicht nur gegen die aktuelle Dringlichkeitsanordnung, sondern auch gegen den Abschnitt des MVG, der die prozeduralen Regelungen hinsichtlich der Strafverfolgung von Regierungsmitgliedern enthielt (Art. 12 bis 24 MVG). Gegen die Anordnung brachte er eine ganze Reihe von Einwänden vor, unter anderem die fehlende Begründung ihres Dringlichkeitscharakters und einen Eingriff in konstitutionelle Befugnisse von Staatsorganen, insbesondere des Staatspräsidenten. Den genannten Abschnitt des MVG hielt Muraru für verfassungswidrig, weil er dem Staatspräsidenten und den beiden Parlamentskammern Verfahrensvorschriften bezüglich der Strafverfolgungsanträge machte, obwohl Art. 109 Abs. 3 Verf. vorsieht, dass das Gesetz über die Ministerielle Verantwortlichkeit lediglich „[d] ie Fälle der Verantwortlichkeit der Regierungsmitglieder und die gegen sie zu verhängenden Strafen“ zu regeln habe. Der Verfassungsgerichtshof kam mit seinem zweiten MVG-Urteil am 25. November 2007 den Forderungen Murarus in wesentlichen Punkten nach: Er hob die gesamte Dringlichkeitsanordnung sowie Art. 16 MVG als verfassungswidrig auf. ${ }^{28}$ Dabei entschied das Gericht, dass die in Art. 109 Abs. 2 Verf. garantierten Entscheidungsrechte des Präsidenten und der Parlamentskammern keinesfalls durch subkonstitutionelle Regelungen beschränkt werden dürften. Daher sei nicht nur die Neuregelung der Präsidialkommission verfassungswidrig, sondern die Einrichtung einer solchen Kommission überhaupt. Dementsprechend wurde neben der Dringlichkeitsanordnung auch der „alte“ Art. 16 MVG kassiert. Die allgemeine Möglichkeit zur Regelung des Verfahrens für die Bearbeitung der Strafverfolgungsanträge wurde vom Verfassungsgerichtshof hingegen als verfassungskonform erachtet.

\section{Die Obstruktionspolitik geht weiter - im Parlament und vor Gericht}

Mit diesem Urteil konnte Präsident Băsescu nun eigenständig über die anhängigen Anträge der Antikorruptionsdirektion entscheiden. Am 16. Januar 2008 genehmigte er die Einleitung aller acht oben genannten Ermittlungsverfahren. ${ }^{29}$ Kurz zuvor war Justizminister Chiuariu aufgrund der gegen ihn erhobenen Korruptionsvorwürfe nach nur neunmonatiger Amtszeit zurückgetreten. ${ }^{30}$ Das Ministerium wurde danach vorübergehend von Verteidigungsminister Teodor Meleşcanu (PNL) geleitet, bevor Ende Februar 2008 der bisherige Wirtschaftsanwalt Cătălin Predoiu (PNL) das Amt übernahm. Die Regierung, die sie stützenden Sozialdemokraten sowie die Konservative Partei führten die Auseinandersetzung

26 Allgemeine Deutsche Zeitung für Rumänien vom 8. Oktober 2007, S. 1.

27 Allgemeine Deutsche Zeitung für Rumänien vom 16. Oktober 2007, S. 1.

28 Verfassungsgerichtsentscheidung Nr. 1133/2007, in: MO 851/2007.

29 Allgemeine Deutsche Zeitung für Rumänien vom 18. Januar 2008, S. 1.

30 Nine O’Clock vom 10. Dezember 2007, S. 1. 
zum Schutz ihrer korruptionsverdächtigen Politiker nun auf anderer Ebene weiter. Zum einen weigerte sich Interims-Justizminister Meleşcanu unter Berufung auf Art. 18 MVG, wonach er "gemäß des Gesetzes“ weiter verfahren sollte, den Antrag des Präsidenten zur Einleitung von Ermittlungen an die Staatsanwaltschaft weiterzuleiten mit der Begründung, dass ihm die kompletten Ermittlungsakten vorzulegen seien. Daraufhin warf nicht nur Băsescu dem Minister Amtsmissbrauch vor - die Exekutive habe schlicht kein Recht, die Aufnahme der Strafverfolgung zu blockieren. Auch die Europäische Kommission forderte Meleşcanu auf, die Zustimmung des Präsidenten der Antikorruptionsdirektion zukommen zu lassen. ${ }^{31}$ Daraufhin gab der amtierende Justizminister seinen Widerstand auf und leitete die Zustimmung des Präsidenten am 22. Januar 2008 an die Staatsanwaltschaft weiter. ${ }^{32}$

Zum anderen verlegten sich PNL, PSD, PC und UDMR auf eine neue Lücke im Gesetz über die Ministerielle Verantwortlichkeit, die erst durch die 2. MVG-Entscheidung des Verfassungsgerichtshofes im November 2007 entstanden war. Mit der Kassation des Art. 16 MVG war nämlich auch die Regelung entfernt worden, derzufolge im Falle einer strafrechtlichen Verfolgung eines Regierungschefs oder Ministers, der zugleich Parlamentsmitglied ist, nach einer Genehmigung durch den Staatspräsidenten auch die jeweilige Parlamentskammer dem Ermittlungsverfahren zuzustimmen hatte. Dadurch entstand nun die Frage, ob nach der neuen Rechtslage die Genehmigung eines der drei Staatsorgane - Abgeordnetenkammer, Senat und Staatspräsident - genügte, oder ob sich diese Organe gegenseitig blockieren konnten. Für ein obligatorisches Zustimmungserfordernis der jeweiligen Parlamentskammer sprachen sich insbesondere deren Präsidenten Bodgan Olteanu (PNL, Abgeordnetenkammer) und Nicolae Văcăroiu (PSD, Senat) aus und wandten sich Anfang Februar 2008 per Organstreitklage an den Verfassungsgerichtshof, um die acht staatsanwaltschaftlichen Ermittlungen erneut zu stoppen. Daraufhin entschied das Gericht am 10. März 2008 in seinem nunmehr dritten MVG-Urteil, dass (1) jedes der drei in Art. 109 Abs. 2 Verf. genannten Staatsorgane ein eigenständiges Recht zur Beantragung einer Strafverfolgung habe, die Organe sich mithin nicht gegenseitig blockieren könnten; (2) infolgedessen für amtierende oder ehemalige Regierungsmitglieder, die zum Zeitpunkt des staatsanwaltschaftlichen Ersuchens auch Mitglieder des Parlaments sind, die jeweilige Parlamentskammer für die Beantragung der Strafverfolgung zuständig sei; und (3) für alle anderen amtierenden oder ehemaligen Regierungsmitglieder nur der Staatspräsident einen Antrag auf Strafverfolgung stellen könne. ${ }^{33}$

Damit konnten vier der acht Strafverfolgungsanträge - die Fälle Zsólt Nagy, Victor Babiuc, Decebal Traian Remeş und Tudor Chiuariu - endlich an die Staatsanwaltschaft weitergeleitet werden. Die anderen vier waren jedoch erneut hinfällig geworden, da der Präsident nun für die Anträge gegen die Abgeordneten Adrian Năstase und Miron Mitrea sowie die Senatoren Codruț Şerȩ̧ und Paul Păcuraru nicht mehr zuständig war.

Darüber hinaus fügte die Abgeordnetenkammer noch am Tag der Verkündung des Verfassungsgerichtsurteils kurz vor der Schlussabstimmung über die Neufassung des Art. 23 Abs. 2 f. MVG, die infolge der ersten MVG-Entscheidung vom Juli 2007 notwendig ge-

31 Allgemeine Deutsche Zeitung für Rumänien vom 22. Januar 2008, S. 1; Stefanie Ricarda Roos, a.a.O. (Fn. 19), S. 2.

32 Allgemeine Deutsche Zeitung für Rumänien vom 24. Januar 2008, S. 1.

33 Verfassungsgerichtsentscheidung Nr. 270/2008, in: MO 290/2008. 
worden war, auch noch einen neuen Art. 16 in das MVG ein. ${ }^{34}$ Danach hätte zukünftig der Justizminister im Falle eines Antrags des Staatspräsidenten auf strafrechtliche Verfolgung eines Ministers oder ehemaligen Regierungsmitglieds, der auch Senator oder Abgeordneter ist, die Zustimmung der jeweiligen Parlamentskammer einholen sollen. Mit „Nein“ votierte neben der rechtsradikalen Partei Großrumänien (Partidul România Mare - PRM) nur die Nachfolgerin der Demokratischen Partei, die Demokratisch-Liberale Partei (Partidul Democrat-Liberal - PD-L). Die PD-L war Ende 2007 aus einer Fusion mit einer Abspaltung der Nationalliberalen hervorgegangen, die im Vorfeld des Auseinanderbrechens der PNL-PD-UDMR-PC-Koalition entstanden war. Mit dem ebenfalls am 10. März 2008 verkündeten dritten MVG-Urteil des Verfassungsgerichtshofes war jedoch bereits offensichtlich, dass diese neuerliche Gesetzesänderung keinen Bestand haben würde, da sie der verfassungsrichterlichen Interpretation des Art. 109 Abs. 2 Verf. explizit widersprach. Daher zog nun die PD-L erfolgreich vor den Verfassungsgerichtshof, der in seinem vierten MVG-Urteil die beschlossene Gesetzesnovelle erwartungsgemäß als verfassungswidrig verwarf. ${ }^{35}$

\section{Verzögerungstaktik: Die Verfolgung der Regierungskorruption hat keine Dringlichkeit}

Damit schien endlich klar zu sein, auf welchem Weg die strafrechtliche Verfolgung auch derjenigen (Ex-)Minister, die aktuell Abgeordnete oder Senatoren waren, korrekt beantragt werden musste. Daher ersuchte die Antikorruptionsdirektion im April 2008 nun das Parlament um Eröffnung staatsanwaltschaftlicher Ermittlungen gegen Năstase, Mitrea, Şereş und Păcuraru. Doch dabei taten sich die nächsten Hürden auf: Die Geschäftsordnungen der beiden Parlamentskammern verlangten für entsprechende Beschlüsse qualifizierte Mehrheiten - der Senat eine absolute Mehrheit seiner Mitglieder und die Abgeordnetenkammer sogar eine Zweidrittelmehrheit. ${ }^{36}$ Zudem machte bereits im Rechtsausschuss der Abgeordnetenkammer eine klare Mehrheit der Parlamentarier deutlich, dass sie nicht gewillt war, die strafrechtliche Verfolgung von Korruptionsdelikten auf höchster Ebene zuzulassen. Gegen die Stimmen der PD-L empfahl der Ausschuss die Ablehnung des Antrags im Fall Năstase. Es handele sich um eine "politically ordered file, aimed at denigrating Nastase“, so der stellvertretende Vorsitzende des Rechtsausschusses Florin Iordache (PSD). ${ }^{37}$ Im Fall Mitrea wurde keine Empfehlung abgegeben. Mitrea selbst bat jedoch im Plenum seine Kollegen um Zustimmung für den Antrag der Antikorruptionsdirektion, allerdings nicht ohne die altbekannten Politisierungsvorwürfe gegen die Staatsanwaltschaft vorzubringen: „Vote for it, please. I want to stand trial, to wrap up there this obvious abuse by the DNA [Antikorruptionsdirektion, Anmerkung des Verfassers]. “38

34 Stenographisches Protokoll der Abgeordnetenkammer vom 10. März 2008, S. 1, http://www. cdep.ro/pls/steno/steno.stenograma?ids=6451\&idm=7 (Abruf am 11. März 2009); Allgemeine Deutsche Zeitung für Rumänien vom 13. März 2008, S. 1.

35 Verfassungsgerichtsentscheidung Nr. 472/2008, in: MO 336/2008; Nine O'Clock vom 19. März 2008, S. 2 und vom 23. April 2008, S. 3.

36 Vgl. Art. 150 Abs. 3 Geschäftsordnung des Senats, in: MO 948/2005 und Art. 155 Abs. 3 Geschäftsordnung der Abgeordnetenkammer, in: MO 35/2006.

37 Nine O'Clock vom 20. Juni 2008, S. 3.

38 Nine O’Clock vom 25. Juni 2008, S. 1. 
Darüber hinaus ließen sich die Parlamentskammern sehr viel Zeit mit der Bearbeitung der Anträge. Erst am 24. Juni 2008 stimmte die Abgeordnetenkammer über die Fälle Năstase und Mitrea ab - scheiterte jedoch bereits am nötigen Quorum von 220 Abgeordneten. Obwohl sich zunächst 248 Parlamentsmitglieder im Saal befunden hatten, gaben nur 208 ihre Stimme ab. ${ }^{39}$ Der Senat hatte über Şereş und Păcuraru überhaupt noch nicht entschieden. Schließlich vertagte das Parlament das Thema auf die Zeit nach der parlamentarischen Sommerpause - die Mehrheiten in den Kammerpräsidien meinten, in der Sache keine Dringlichkeit erkennen zu können. Dies änderte sich erst, als die EU-Kommission in ihrem Fortschrittsbericht Ende Juli 2008 die Haltung des Parlaments harsch kritisierte:

„Das Versagen bei der Weiterverfolgung dieser Fälle macht die positiven Bemühungen bei den Ermittlungen zunichte. [...] Die Bemühungen der Behörde [Antikorruptionsdirektion, Anmerkung des Verfassers] um eine Fortsetzung der Untersuchungen in einigen wichtigen Fällen waren jedoch bisher fruchtlos, da das Parlament die Empfehlung zur Einleitung von Gerichtsverfahren versagte. Die Abneigung der Justiz und des Parlaments, Ermittlungen in diesen prominenten Fällen zu genehmigen, hat zu einem Vertrauensverlust in der Öffentlichkeit geführt. [...] Unabhängige Ermittlungen gegen frühere Regierungsmitglieder und Parlamentsabgeordnete müssen fortgesetzt werden dürfen, damit das Vertrauen der Öffentlichkeit in die Korruptionsbekämpfung wiederhergestellt und der Respekt vor dem Gesetz gestärkt wird." ${ }^{40}$

Daraufhin konnte sich Traian Băsescu mit seiner bereits einen Monat zuvor erhobenen Forderung nach außerordentlichen Sitzungen der Parlamentskammern durchsetzen. ${ }^{41}$ Doch auch der durch den EU-Bericht aufgebaute Druck genügte nicht, um die Parlamentarier zu einer durchgängigen Beendigung ihrer Blockade der Korruptionsbekämpfung zu bewegen. In der Abgeordnetenkammer erreichte am 13. August 2008 keiner der beiden Anträge die erforderliche Zweidrittelmehrheit von 218 Stimmen: Im Fall Năstase stimmten 120 Abgeordnete für und 150 gegen die Strafverfolgung bei sechs ungültigen Voten; im Fall Mitrea gab es 160 Ja- und 115 Nein-Stimmen bei einem ungültigen Votum. ${ }^{42}$ Damit mussten beide Verfahren vorläufig eingestellt werden. Miron Mitrea legte jedoch am 3. September 2008 sein Parlamentsmandat nieder und ermöglichte so den Beginn der staatsanwaltschaftlichen Ermittlungen. ${ }^{43}$ Der Senat erreichte hingegen bei den Anträgen gegen Codruț Şereş mit 79 zu 40 Stimmen beziehungsweise Paul Păcuraru mit 75 zu 44 Stimmen bei jeweils einem ungültigen Votum die notwendigen absoluten Mehrheiten. Arbeitsminister Păcuraru wurde zudem am 22. September 2008 von Präsident Băsescu entsprechend der obligatorischen Verfassungsvorschriften (Art. 109 Abs. 3 Verf.) des Amtes enthoben, nachdem die Staatsanwaltschaft gegen ihn Anklage erhoben hatte. ${ }^{44}$

39 Allgemeine Deutsche Zeitung für Rumänien vom 26. Juni 2008, S. 1.

40 Kommission der Europäischen Gemeinschaften, Fortschritte Rumäniens im Rahmen des Kooperations- und Kontrollverfahrens, Brüssel, 23. Juli 2008, S. 5 f., http://ec.europa.eu/dgs/secretariat_general/cvm/docs/romania_report_20080723_de.pdf (Abruf am 9. März 2009).

41 Vgl. Nine O'Clock vom 27. Juni 2008, S. 1 und vom 3. Juli, S. 3.

42 Stenographisches Protokoll der Abgeordnetenkammer vom 13. August 2008, S. 1, http://www. cdep.ro/pls/steno/steno.stenograma?ids=6512\&idm=10\&idl=1 (Abruf am 11. März 2009); Nine O'Clock vom 14. August 2008, S. 1.

43 Nine O’Clock vom 15. August und 4. September 2008, S. 3. Mitrea kehrte Ende November nach den Wahlen als Senator ins Parlament zurück.

44 Stenographisches Protokoll des Senats vom 26. August 2008, S. 1, http://www.cdep.ro/pls/steno/ steno.stenograma?ids=6516\&idm=4\&idl=1 (Abruf am 11. März 2009); Allgemeine Deutsche Zeitung für Rumänien vom 28. August und 24. September 2008, S. 1. 
Damit blieb von den acht ehemaligen Regierungsmitgliedern vor staatsanwaltschaftlichen Ermittlungen nur noch Adrian Năstase geschützt, der als Präsident des Nationalrates der PSD unterdessen in die Führungsgremien der Sozialdemokraten hatte zurückkehren können. Anfang September hatte die Antikorruptionsdirektion zwei weitere Anträge gegen ihn bei der Abgeordnetenkammer eingereicht, die sich auf den Fall "Tamara“ sowie den Vorwurf der Annahme von Bestechungsgeldern beziehungsweise Geschenken für die Vergabe öffentlicher Ämter bezogen. Ein letztes Mal unternahm eine Mehrheit aus PSD, PNL und PRM im Rechtsausschuss einen Versuch, die Strafverfolgung des früheren Premierministers zu verhindern. Sie sandte kurzerhand die staatsanwaltschaftlichen Ersuchen an die Direktion mit der lapidaren Begründung zurück, „the existing evidence is null“ 45 . Diese offensichtliche Gesetzesübertretung wurde zwar seitens der PD-L scharf kritisiert, denn weder die Verfassung noch das Gesetz über die Ministerielle Verantwortlichkeit sahen eine solche Möglichkeit ohne einen Beschluss des Plenums vor. Doch es blieb bis zum Ende der Wahlperiode bei diesem Ergebnis. Allerdings hatten bereits Anfang Juli 2008 die Abgeordneten und Senatoren der PD-L vor dem Verfassungsgerichtshof gegen die Artikel der parlamentarischen Geschäftsordnungen geklagt, die die erhöhten Mehrheiten für die Genehmigung der Strafverfolgung gegen ehemalige Minister vorsahen. Dabei bezogen sie sich vor allem auf Art. 76 Abs. 2 Verf., der für Beschlüsse des Parlaments eindeutig das Erfordernis „der Mehrheit der in jeder Kammer anwesenden Mitglieder" festlegte. Der Verfassungsgerichtshof kam den Anträgen der Demokratisch-Liberalen Partei am 1. Oktober 2008 in vollem Umfang nach und verkündete damit sein fünftes MVG-Urteil innerhalb von nur 15 Monaten. ${ }^{46}$ Mit diesem Erfolg konnte sich die PD-L zudem endgültig als „die einzige Partei, die gegen die Korruption kämpft“, stilisieren. ${ }^{47}$

Nun schienen alle denkbaren verfassungs- und verfahrensrechtlichen Möglichkeiten ausgereizt. Der zwei Jahre andauernde Konflikt um die strafrechtliche Verantwortlichkeit von Regierungsmitgliedern fand jedoch erst nach den Parlamentswahlen vom 30. November 2008 und der Bildung einer Koalition aus PD-L, PSD und PC ein vorläufiges Ende. Die Wahlen hatten einen knappen Sieg der Allianz PSD+PC vor der PD-L, der Nationalliberalen Partei und dem UDMR gebracht, wobei die PD-L aufgrund einiger Anomalien des neu eingeführten Mehrheitswahlsystems in Einerwahlkreisen mit proportionaler Verteilung der Reststimmen trotz ihrer knappen Niederlage in beiden Kammern mehr Mandate erhielt als PSD+PC. ${ }^{48}$ Der von Traian Băsescu nominierte Premierminister Emil Boc (PD-L) bildete eine Koalitionsregierung mit den Sozialdemokraten und der Konservativen Partei - anstatt mit der inhaltlich viel näher stehenden PNL zusammenzugehen. Eine solche Koalition schien jedoch nach dem Bruch des PNL-PD-Bündnisses 2007 aus Sicht der DemokratischLiberalen und insbesondere des Staatspräsidenten schlicht ausgeschlossen.

45 Nine O’Clock vom 11. September 2008, S. 3.

46 Verfassungsgerichtsentscheidungen Nr. 989/2008 und Nr. 990/2008, in: MO 716/2008.

47 So der Vorsitzende des Bukarester Stadtverbands der PD-L und Bürgermeister eines Hauptstadtbezirks, Liviu Negoiță, in: Allgemeine Deutsche Zeitung für Rumänien vom 3. Oktober 2008, S. 1.

48 Vgl. Anneli Ute Gabanyi, Die Parlamentswahlen 2008 in Rumänien, in: Südosteuropa Mitteilungen, 49. Jg. (2009), H. 1, S. 64 - 75. Das Wahlergebnis im Detail: Allianz PSD+PC 33,1 Prozent (Abgeordnetenkammer) / 34,2 Prozent (Senat); PD-L 32,4 / 33,6 Prozent; PNL 18,6 / 18,8 Prozent; UDMR: 6,2 / 6,4 Prozent. Die PRM scheiterte mit 3,2 / 3,6 Prozent erstmals seit 1992 ebenso an der Fünfprozenthürde wie die PNG-CD George Becalis mit 2,3 / 2,5 Prozent. 
In der Folge gelang es den Sozialdemokraten im Rechtsausschuss der Abgeordnetenkammer Anfang Februar 2009 zwar nochmals, mit zehn zu neun Stimmen gegen ihren Koalitionspartner dem Plenum der Kammer eine Ablehnung der neuerlichen Anträge der Staatsanwaltschaft gegen Năstase zu empfehlen und darüber hinaus von der Antikorruptionsdirektion weitere Unterlagen anzufordern. ${ }^{49}$ Doch angesichts des geänderten rechtlichen Rahmens für die Abstimmung in der Abgeordnetenkammer und der neuen Mehrheitsverhältnisse im Parlament gab Năstase kurz darauf selbst den Widerstand gegen seine strafrechtliche Verfolgung auf, allerdings nicht ohne Băsescu die Politisierung der Judikative vorzuwerfen. ${ }^{50}$ Mit 158 zu 128 Stimmen bei zwei ungültigen Voten beantragte die Abgeordnetenkammer am 4. März 2009 schließlich die Eröffnung staatsanwaltschaftlicher Ermittlungen gegen Adrian Năstase. ${ }^{51}$

\section{Ausblick: Perspektiven der Korruptionsbekämpfung in Rumänien}

Mehr als zwei Jahre lang hat eine „Koalition der Unwilligen“52 in der rumänischen politischen Elite gegen die Verfolgung allfälliger Korruptionsdelikte von Regierungsmitgliedern gekämpft. Nun liegen die in Rede stehenden Fälle in den Händen der Justiz. Bisher ist zwar noch keiner der betroffenen Politiker verurteilt worden, aber gegen die Mehrzahl wurde mittlerweile zumindest Anklage erhoben. ${ }^{53}$ Die Liste der korruptionsverdächtigen Spitzenpolitiker ist seitdem jedoch keineswegs kürzer geworden. Im Gegenteil: Im Sommer 2009 beantragte die Staatsanwaltschaft gegen zwei weitere Ex-Minister die Eröffnung der Strafverfolgung. Dem ehemaligen Privatisierungsminister Ovidiu Muşetescu (2001 bis 2003, PSD) wird Bestechlichkeit beim Verkauf von Staatsunternehmen vorgeworfen, und die ExJugend- und Sportministerin Monica Iacob-Ridzi (2008/2009, PD-L) muss sich wegen angeblicher Unregelmäßigkeiten bei Auftragsvergaben verantworten. Iacob-Ridzi war aufgrund dieser Vorwürfe erst im Juli 2009 zurückgetreten. ${ }^{54}$ Staatspräsident Băsescu kam dem Antrag der Staatsanwaltschaft gegen $M$ usetescu ebenso nach, wie die Abgeordnetenkammer den Antrag gegen Iacob-Ridzi mit 192 zu 35 Stimmen billigte. Dabei zeigte sich, dass sich die PD-L im Gegensatz zu den anderen Parteien nicht der Verfolgung von Straftaten ihrer eigenen Mitglieder verweigerte.

49 Nine O'Clock vom 5. Februar 2009, S. 2.

50 Nine O'Clock vom 5. März 2009, S. 1; vgl. Allgemeine Deutsche Zeitung für Rumänien vom 6. März 2009, S. 1.

51 Stenographisches Protokoll der Abgeordnetenkammer vom 4. März 2009, http://www.cdep.ro/ $\mathrm{pls} / \mathrm{steno} /$ steno.stenograma?ids=6591\&idm=3\&idl=1 (Abruf am 12. März 2009).

52 So die treffende Formulierung bei Alina Mungiu-Pippidi, The Learning Curve Revisited. Building the Rule of Law in East Central Europe, 5th ECPR General Conference, Potsdam, 10. bis 12. September 2009, S. 18.

53 Vgl. die Websites der Antikorruptionsdirektion, http://www.pna.ro (Abruf am 1. Oktober 2009), und des Hohen Kassations- und Gerichtshofes, http://www.scj.ro (Abruf am 1. Oktober 2009).

54 Allgemeine Deutsche Zeitung für Rumänien vom 1. Juli 2009, S. 1; Nine O’Clock vom 23. Juli 2009, S. 1. Darüber hinaus warfen PNL und PSD einer weiteren PD-L-Politikerin, der Tourismusministerin Elena Udrea Amtsmissbrauch, Interessenkonflikte und die Vernachlässigung von Dienstpflichten vor und strengten im Parlament ein Verfahren gegen sie an, bis Ende September 2009 jedoch noch ohne Ergebnisse; vgl. Allgemeine Deutsche Zeitung für Rumänien vom 25. September 2009, S. 1. 
Zudem gab es 2009 eine erste Verurteilung in einem Fall von Regierungskorruption, wenn auch nur aus der „zweiten Reihe“: Der amtierende Staatssekretär für die Probleme der „Revolutionäre von 1989“, Emilian Cutean (PSD), wurde im Juni erstinstanzlich zu einer Freiheitsstrafe von fünf Jahren ohne Bewährung verurteilt. Er hatte dieses Amt bereits während der PSD-PC-Regierung 2000 bis 2004 inne gehabt. Kurz vor Ende seiner damaligen Amtszeit hatte er ca. 80.000 Euro veruntreut und damit unter anderem seine Privatwohnung finanziert. ${ }^{55}$ Trotz des laufenden Verfahrens hatte die PSD bei den Koalitionsverhandlungen mit der PD-L Ende 2008 Cuteans erneute Ernennung in die Regierung durchgesetzt. Nach der Verkündung des Urteils wurde er jedoch von Premierminister Boc (PD-L) entlassen.

Gleichwohl wäre es naiv anzunehmen, dass nun alle Hindernisse für eine wirkungsvolle Bekämpfung der großen politischen Korruption ausgeräumt seien. Vielmehr ist auch zukünftig eine Reihe von Problemen zu erwarten. Das erste ist dabei grundlegender Natur: Wie die Analyse der Auseinandersetzungen um Korruptionsstrafverfahren gegen Regierungsmitglieder gezeigt hat, ist das Thema Korruption in Rumänien einer starken politischen Instrumentalisierung ausgesetzt. Dies wird nicht zuletzt daran deutlich, dass nahezu täglich Korruptionsvorwürfe gegen Mitglieder von jeweils anderen Parteien öffentlich vorgebracht werden. Dabei entsteht der Eindruck, dass es rumänischen Politikern primär um die Schwächung des politischen Gegners und nicht um die Lösung des Problems Korruption geht. Dies kann letztlich auch nicht weiter verwundern - angesichts der Tatsache, dass Korruption offensichtlich über alle Parteigrenzen hinweg in der gesamten politischen Elite des Landes weit verbreitet ist.

Zweitens bleibt das Grundübel des parlamentarischen Zustimmungserfordernisses für eine Strafverfolgung von Regierungsmitgliedern bestehen. Dessen Folgen zeigten sich bereits im Juni 2009 wieder, als auf der Tagesordnung der Abgeordnetenkammer erneut diejenigen Anträge der Antikorruptionsdirektion gegen Adrian Năstase standen, die im Vorjahr abgelehnt worden waren. Mit dem fadenscheinigen Argument, die Staatsanwaltschaft könne keine „Wiederholungsabstimmung“ über dieselben Anträge erbeten, lehnte die Kammer den Antrag mit 116 zu 100 Stimmen bei zehn Enthaltungen gegen die Stimmen der PD-L ab. ${ }^{56}$ Anfang Oktober 2009 zerbrach schließlich die von Beginn an instabile PD-L-PSDPC-Koalition, wobei die Bildung einer neuen Regierungsmehrheit im Parlament in den Wochen danach nicht absehbar war. ${ }^{57}$ Neue Mehrheiten für allfällige Strafverfolgungsanträge scheinen damit ebenfalls in weite Ferne gerückt.

Drittens kann es als nahezu sicher gelten, dass die Betroffenen auch zukünftig weitere Gesetzeslücken auftun und verfahrenstechnische Verzögerungsmöglichkeiten nutzen werden. Der bisher äußerst schleppende Verlauf der ersten Verfahren gegen Năstase bestätigt diese Erwartung. ${ }^{58}$

Viertens sind die rumänischen Gerichte bisher nicht gerade dadurch aufgefallen, in Korruptionsverfahren besonders harte Urteile zu fällen. Zwar sieht der in den vergangenen Jahren geschaffene Rechtsrahmen im europäischen Vergleich zum Teil gravierende Höchststrafen vor. ${ }^{59}$ Gleichwohl kommt es in der Praxis zumeist nur zu Gefängnisstrafen von

55 Allgemeine Deutsche Zeitung für Rumänien vom 17. Juni 2009, S. 1.

56 Nine O'Clock vom 23. Juni 2009, S. 1.

57 Allgemeine Deutsche Zeitung für Rumänien vom 2. Oktober 2009, S. 1.

58 Vgl. Nine O’Clock vom 16. Juni 2009, S. 2.

59 Vgl. die ausführliche Darstellung bei Hildebrand, a.a.O. (Fn. 23), S. 278 ff. 
durchschnittlich ein bis zwei Jahren, die in der Regel auch noch zur Bewährung ausgesetzt werden. ${ }^{60}$ Solche Strafen sind nicht dazu angetan, eine „abschreckende und damit präventive Wirkung" zu entfalten, wie die Europäische Kommission bereits in ihrem ersten Fortschrittsbericht nach dem EU-Beitritt Rumäniens im Juni 2007 zu Recht kritisierte. ${ }^{61}$ Es ist nicht zu erwarten, dass sich diese Rechtsprechungspraxis in naher Zukunft grundlegend ändern wird.

Fünftens schließlich besteht für all diejenigen korruptionsverdächtigen Politiker, die aktuell als Parlamentarier tätig sind, ein weiterer Schutzmechanismus: ihre parlamentarische Immunität. Wie eingangs bereits erwähnt, dürfen sie ohne die Zustimmung ihrer Kammer „nicht durchsucht, festgehalten oder verhaftet werden“ (Art. 72 Abs. 1 Verf.). Ein eventueller Vollzug von Gefängnisstrafen läge also erneut in der politischen Entscheidungsbefugnis des Parlaments, und es dürfte interessant sein zu sehen, ob die Legislative tatsächlich einer Inhaftierung prominenter Parlamentarier zustimmen würde. Gegenwärtig genießen vier der ehemaligen Regierungsmitglieder diesen Schutz: die Abgeordneten Tudor Chiuariu, Monica Iacob-Ridzi und Adrian Năstase sowie der Senator Miron Mitrea. Zu diesen ehe-maligen Premierministern beziehungsweise Ministern kommen derzeit acht (!) weitere Mitglieder des rumänischen Parlaments hinzu, gegen die wegen anderweitiger Korruptionsvorwürfe ermittelt wird. Die Tageszeitung Cotidianul witzelte daher nach den Parlamentswahlen vom November 2008: „Antikorruptionsdirektion hat eigene Parlamentsfraktion“62. Ob die Bekämpfung der „großen“ politischen Korruption in Rumänien daher in nächster Zeit tatsächlich erste greifbare Erfolge zeitigen wird, muss weiterhin skeptisch eingeschätzt werden.

60 Der Fall Cutean ist bisher eine seltene Ausnahme und zudem noch nicht letztinstanzlich entschieden.

61 Kommission der Europäischen Gemeinschaften, Bericht über Rumäniens Fortschritte bei den Begleitmaßnahmen nach dem Beitritt, Brüssel, 27. Juni 2007, S. 19, http://ec.europa.eu/dgs/secretariat_general/cvm/docs/romania_report_20070627_de.pdf (Abruf am 9. März 2009). Allerdings drängt sich beispielsweise aus bundesdeutscher Perspektive die Frage auf, ob hinsichtlich der Strafmaße tatsächlich ein Unterschied zu den „konsolidierten“ Rechtsstaaten Mittel- und Westeuropas besteht, wie es der Fortschrittsbericht impliziert.

62 Cotidianul vom 2. Dezember 2008, http://www.cotidianul.ro/dna_are_grup_parlamentar-66471. html (Abruf am 15. März 2009). Einer dieser acht Parlamentarier, der neu gewählte Abgeordnete Virgil Pop (PNL), befand sich zum Zeitpunkt der Wahlen sogar in Untersuchungshaft.

\section{Das zyprische Repräsentantenhaus zwischen Verfassungsvorgaben und Teilungsnöten: Entwicklungen seit 1960}

\section{Dimitrios Parashu}

Die Republik Zypern, seit dem 1. Mai 2004 eines der jüngeren Mitglieder der Europäischen Union, hat nicht erst seit ihrer Unabhängigkeit vom Vereinigten Königreich 1960 eine stürmische Geschichte hinter sich. Man weiß, dass die Insel seit dem Sommer 1974 de facto in zwei geographische Hälften geteilt ist: Den (international als Republik Zypern anerkannten) griechischen Süden und den (nur von Ankara als eigenständige „Republik“ ge- 